\title{
AN EXACTLY SOLVABLE MODEL FOR THE INTERACTION OF LINEAR WAVES WITH KORTEWEG-DE VRIES SOLITONS*
}

\author{
P. D. MILLER ${ }^{\dagger}$ AND S. R. CLARKE
}

\begin{abstract}
Under certain mode-matching conditions, small-amplitude waves can be trapped by coupling to solitons of nonlinear fields. We present a model for this phenomenon, consisting of a linear equation coupled to the Korteweg-de Vries (KdV) equation. The model has one parameter, a coupling constant $\kappa$. For one value of the coupling constant, $\kappa=1$, the linear equation becomes the linearized $\mathrm{KdV}$ equation, for which the linear waves can indeed be trapped by solitons and, moreover, for which the initial value problem for the linear waves has been solved exactly by Sachs [S83] in terms of quadratic forms in the Jost eigenfunctions of the associated Schrödinger operator. We consider in detail a different case of weaker coupling, $\kappa=1 / 2$. We show that in this case linear waves may again be trapped by solitons, and like the stronger coupling case $\kappa=1$, the initial value problem for the linear waves can also be solved exactly, this time in terms of linear forms in the Jost eigenfunctions. We present a family of exact solutions, and we develop the completeness relation for this family of exact solutions, finally giving the solution formula for the initial value problem. For $\kappa=1 / 2$, the scattering theory of linear waves trapped by solitons is developed. We show that there exists an explicit increasing sequence of bifurcation values of the coupling constant, $\kappa=1 / 2,1,5 / 3, \ldots$, for which some linear waves may become trapped by solitons. By studying a third-order eigenvalue equation, we show that for $\kappa<1 / 2$ all linear waves are scattered by solitons, and that for $1 / 2<\kappa<1$, as well as for $\kappa>1$, some linear waves are amplified by solitons.
\end{abstract}

Key words. solitons, Korteweg-de Vries equation, coupled systems, completeness relations, wave trapping

AMS subject classifications. 37K40, 35Q53, 42A65

PII. S0036141099365431

1. Introduction. This paper is concerned with solving the coupled system of equations

$$
\begin{aligned}
& \partial_{t} A+\partial_{x}\left[\frac{1}{2} A^{2}+\partial_{x}^{2} A\right]=0, \\
& \partial_{t} B+\partial_{x}\left[\kappa A B+\partial_{x}^{2} B\right]=0,
\end{aligned}
$$

where $\kappa$ is a real parameter. Of course, the nonlinear equation for $A(x, t)$ is simply the Korteweg-de Vries (KdV) equation, and it can be solved independently by the inverse-scattering transform [GGKM67]. The coupled system (1) and (2) is a partially linearized version of the system proposed by Hirota and Satsuma [HS81] as a model for the dynamics of coupled long waves.

The coupled system (1) and (2) can be solved exactly when $\kappa=1$ and when $\kappa=1 / 2$. The case of $\kappa=1$ is well known, for then the equation (2) is just the $\mathrm{KdV}$ equation itself linearized about the solution $A(x, t)$. An elementary exact solution of the linear equation (2) in this case is given by $B(x, t)=\partial_{x} A(x, t)$. Further solutions can be expressed in terms of derivatives of the squared eigenfunctions of the related Schrödinger operator with potential $A$ [S83].

*Received by the editors December 7, 1999; accepted for publication November 27, 2000; published electronically June 8, 2001.

http://www.siam.org/journals/sima/33-2/36543.html

${ }^{\dagger}$ Department of Mathematics, University of Michigan, East Hall, 525 East University Avenue, Ann Arbor, MI 48109 (millerpd@umich.edu).

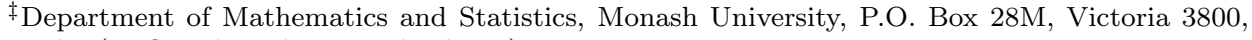
Australia (src@mail.maths.monash.edu.au). 
The case of $\kappa=1 / 2$ is essentially different. In this case, the linear equation (2) is no longer the linearization of KdV about any solution. An elementary exact solution of the linear equation in this case is given simply by $B(x, t)=A(x, t)$. The main goal of this paper is to construct the general solution of the initial value problem for this linear equation when $A(x, t)$ is a multisoliton solution of $\mathrm{KdV}$.

One way to make clear the difference between the cases $\kappa=1$ and $\kappa=1 / 2$ is to consider $A(x, t)$ to be the simple soliton solution of $\mathrm{KdV}(1)$ :

$$
A(x, t)=12 \eta^{2} \operatorname{sech}^{2}\left(\eta\left(x-4 \eta^{2} t-\alpha\right)\right)=-V(\chi),
$$

where $\chi=x-c t-\alpha$ and the velocity is $c=4 \eta^{2}$. If we look for solutions of the linear equation (2) that are traveling waves with speed $c$, we find the equation

$$
\left[-\kappa V(\chi) B(\chi)+B^{\prime \prime}(\chi)\right]^{\prime}=c B^{\prime}(\chi) .
$$

Integrating once, using vanishing boundary conditions at $\chi= \pm \infty$, yields a Schrödinger eigenvalue problem for $B$ :

$$
-B^{\prime \prime}(\chi)+\kappa V(\chi) B(\chi)=E B(\chi),
$$

where $E=-c$. For $\kappa=1 / 2$, it follows from the fact that $B(x, t)=A(x, t)$ is a solution of (2) that the function $B(\chi)=V(\chi)$ is an eigenfunction of the Schrödinger operator with eigenvalue $E=-c=-4 \eta^{2}$. Since it has no zeros, it is the ground state eigenfunction. We will see below that there is also one excited state for $\kappa=1 / 2$, although it is not relevant here since it corresponds to a different velocity. On the other hand, for $\kappa=1, B(x, t)=\partial_{x} A(x, t)$ is a solution of $(2)$, which implies that the function $B(\chi)=\partial_{x} V(\chi)$ is an eigenfunction of the Schrödinger operator with the same eigenvalue $E=-c=-4 \eta^{2}$. In this case, the eigenfunction has a single zero and therefore is the first excited state. It follows that there are at least two eigenvalues for $\kappa=1$. In fact, there are exactly three states in this case. A final observation is that from the construction of the one-soliton solution of KdV (see (8), (9), and (10) below) it follows that for $\kappa=1 / 6$, the function $B(\chi)=V(\chi)$ is an eigenfunction of the Schrödinger operator with eigenvalue $E=-c / 4=-\eta^{2}$. It is the ground state and the only eigenfunction. These relationships are summarized in Figure 1.1. We will have more to say about this picture when we discuss the trapping of linear waves by solitons for general values of $\kappa$ in section 6 .

The rest of this paper is primarily concerned with developing the general solution of the initial value problem for (2) with $\kappa=1 / 2$ when $A(x, t)$ is an $N$-soliton solution of $\mathrm{KdV}(1)$. In section 2 we show how for $\kappa=1 / 2$ a large family of exact solutions of (2) can be obtained from the simultaneous solutions of the Lax pair for KdV. When the solution of $\mathrm{KdV}$ contains only solitons and no radiation, the construction of Lax eigenfunctions is completely algorithmic and algebraic, and consequently the corresponding family of solutions of (2) for $\kappa=1 / 2$ can be obtained with great practicality. In section 3 we then establish that in the $N$-soliton case there are enough of these exact solutions of (2) for $\kappa=1 / 2$ to expand for fixed $t$ any absolutely continuous $L^{1}(\mathbb{R})$ function of $x$. This fact then leads to a general solution formula for (2) simply by expanding the initial data. We present and discuss this formula in section 4 . There will turn out to be $N$ linearly independent solutions that are asymptotically confined to the union of soliton trajectories and can therefore be considered to be bound states. In section 5 we compute the scattering matrix that relates the asymptotic behavior of bound states for $t \rightarrow-\infty$ to the corresponding behavior for $t \rightarrow+\infty$. In section 6 we 


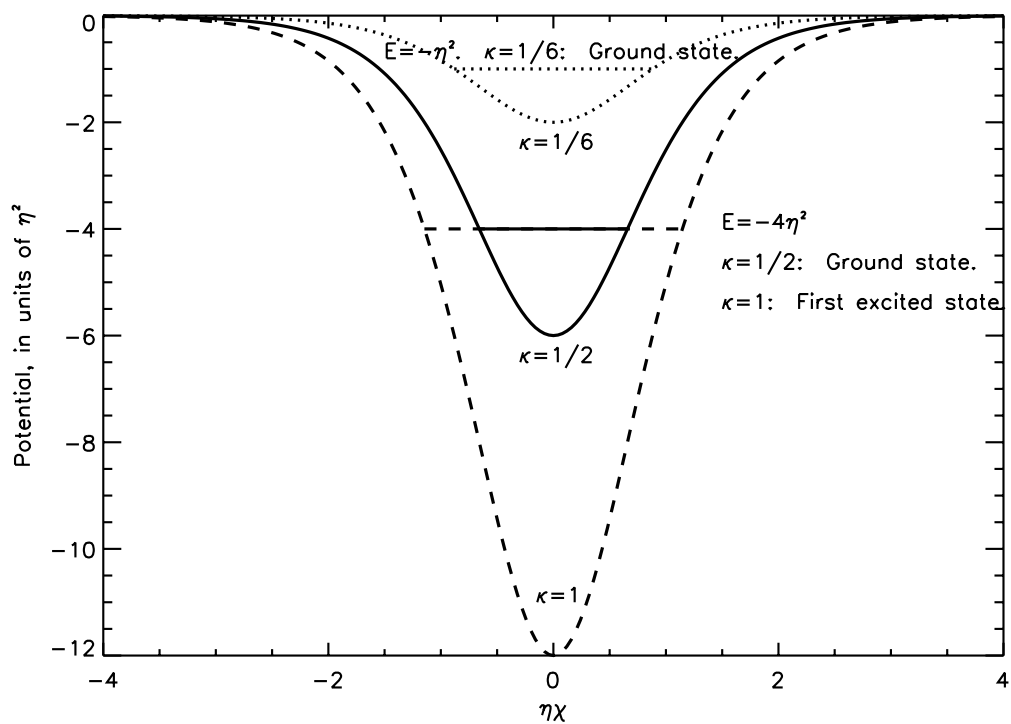

FIG. 1.1. Energy levels of the $12 \kappa \eta^{2} \operatorname{sech}^{2}(\eta \chi)$ potential for three different values of the coupling constant $\kappa$.

consider general values of the coupling constant $\kappa$ and describe the behavior of some solutions of (2) when $A(x, t)$ is a one-soliton solution of $\mathrm{KdV}(1)$. These calculations indicate the exceptional nature of the two values $\kappa=1 / 2$ and $\kappa=1$. In the appendix, we describe several physical applications of the coupled system (1) and (2) to topics in molecular dynamics, mechanics, soliton theory, and the fluid dynamics of internal waves.

2. Exact solution formulas for $\kappa=\mathbf{1 / 2}$. As is well known [GGKM67], the $\mathrm{KdV}$ equation (1) is the compatibility condition for a pair of linear equations involving a complex parameter $\lambda$ for an auxiliary function $f(x, t, \lambda)$. This pair of linear equations is

$$
\partial_{x}^{2} f=-\frac{\lambda^{2}}{4} f-\frac{1}{6} A f \quad \text { and } \quad \partial_{t} f=\frac{1}{6} \partial_{x} A \cdot f+\left(\lambda^{2}-\frac{1}{3} A\right) \partial_{x} f
$$

and is called a Lax pair. A simultaneous solution $f(x, t, \lambda)$ of these linear equations exists if and only if the function $A(x, t)$ satisfies $\mathrm{KdV}(1)$. Suppose that this is the case. Then, it is a direct matter to verify that for fixed but arbitrary $\lambda \in \mathbb{C}$, the two functions defined by

$$
B(x, t):=\partial_{x}\left[f(x, t, \lambda) \exp \left( \pm \frac{i}{2}\left(\lambda x+\lambda^{3} t\right)\right)\right]
$$

are solutions of the linear equation (2) when $\kappa=1 / 2$. Note here an important point of departure from the other solvable case, namely, $\kappa=1$, where (2) is the linearized $\mathrm{KdV}$ equation. In the latter case, particular solutions are expressed in terms of the $x$-derivative of the square of the Lax eigenfunction $f(x, t, \lambda)$ [GGKM74, S83]. By contrast, the formula (7) for solutions of (2) for $\kappa=1 / 2$ is linear in $f(x, t, \lambda)$. This fact leads to some important simplifications. 
The formula (7) is only really practical to use if one can explicitly compute the function $f(x, t, \lambda)$. This will be the case if the solution $A(x, t)$ of $\mathrm{KdV}(1)$ is a pure $N$-soliton solution. For each fixed $t, A(x, t)$ is then a reflectionless potential of the Schrödinger equation in the Lax pair (6). The multisoliton solutions of KdV and the associated solutions of the Lax pair are constructed as follows [KM56]. Let $f_{+}(x, t, \lambda)$ be given by

$$
f_{+}(x, t, \lambda):=\left(1+\sum_{n=0}^{N-1} \lambda^{n-N} f_{n}(x, t)\right) \exp \left(-\frac{i}{2}\left(\lambda x+\lambda^{3} t\right)\right),
$$

where the $f_{n}(x, t)$ are unknown coefficients. Choose $N$ positive numbers $\eta_{1}>\eta_{2}>$ $\cdots>\eta_{N}$, and $N$ arbitrary real numbers $\alpha_{1}, \ldots, \alpha_{N}$, and insist that $f_{+}(x, t, \lambda)$ satisfy the relations

$$
f_{+}\left(x, t, 2 i \eta_{n}\right)=(-1)^{n+1} \exp \left(2 \eta_{n} \alpha_{n}\right) f_{+}\left(x, t,-2 i \eta_{n}\right)
$$

for all $n=1, \ldots, N$. It is easy to see that these relations imply a square linear algebraic system for the coefficients $f_{n}(x, t)$. The determinant of the system is always nonzero, and so the coefficients $f_{n}(x, t)$ are determined uniquely from the soliton eigenvalues $\left\{\lambda_{n}=2 i \eta_{n}\right\}$ and the norming constants $\left\{\alpha_{n}\right\}$ in terms of exponential functions. From this construction, it can be shown that if one chooses

$$
A(x, t):=6 i \partial_{x} f_{N-1}(x, t),
$$

then $f_{+}(x, t, \lambda)$ and $f_{-}(x, t, \lambda):=f_{+}(x, t,-\lambda)$ are two simultaneous solutions of the Lax pair (6), and the function $A(x, t)$ defined by (10) satisfies KdV (1). The two functions $f_{ \pm}(x, t, \lambda)$ are linearly independent for all nonzero $\lambda \neq \pm 2 i \eta_{n}$. According to the linear relations (9) that determine the coefficients, at the exceptional values of $\lambda$ the two functions are proportional.

The solution $A(x, t)$ of $\mathrm{KdV}$ so constructed represents the interaction of $N$ solitons. In particular, as $t \rightarrow \pm \infty$, the solution can be represented in the form

(11) $A(x, t) \sim \sum_{n=1}^{N} A_{n}^{ \pm}(x, t), \quad$ where $\quad A_{n}^{ \pm}(x, t):=12 \eta_{n}^{2} \operatorname{sech}^{2}\left(\eta_{n}\left(x-\alpha_{n}^{ \pm}\right)-4 \eta_{n}^{3} t\right)$,

where the asymptotic phase constants $\alpha_{n}^{ \pm}$are functions of the $\eta_{n}$ and $\alpha_{n}$.

Below we will need the asymptotic behavior of the functions $f_{ \pm}(x, t, \lambda)$ as $x \rightarrow$ $\pm \infty$ for $\lambda$ and $t$ fixed. It can be shown that the coefficient functions $f_{n}(x, t)$ remain bounded as $x \rightarrow \pm \infty$. Then, letting $x$ tend to $\pm \infty$ in the linear relations (9), one finds from dominant balance arguments that

$$
\left.\lim _{x \rightarrow \pm \infty}\left(1+\sum_{n=0}^{N-1} \lambda^{n-N} f_{n}(x, t)\right)\right|_{\lambda= \pm 2 i \eta_{n}}=0 .
$$

These relations imply that

$$
\lim _{x \rightarrow \pm \infty}\left(1+\sum_{n=0}^{N-1}( \pm \lambda)^{n-N} f_{n}(x, t)\right)=\lambda^{-N} \prod_{n=1}^{N}\left(\lambda-2 i \eta_{n}\right) .
$$

Therefore, for all $\lambda \in \mathbb{C}$,

$$
\lim _{x \rightarrow \pm \infty} f_{+}(x, t, \lambda) \exp \left(\frac{i}{2}\left(\lambda x+\lambda^{3} t\right)\right)=\lambda^{-N} \prod_{n=1}^{N}\left(\lambda \mp 2 i \eta_{n}\right) .
$$


The large $|x|$ asymptotics for the other solution $f_{-}(x, t, \lambda)$ follow from the definition $f_{-}(x, t, \lambda)=f_{+}(x, t,-\lambda)$. From these asymptotics, it is easy to construct the appropriate linear combinations of $f_{ \pm}(x, t, \lambda)$ that correspond to the Jost functions of the Schrödinger equation, normalized at $x= \pm \infty$.

In the formula (7) we have a choice of sign in the exponent. In fact, it is easy to see that if one considers the totality of solutions obtained for all complex $\lambda$, the choice of sign is redundant. In what follows, we adopt a particular choice of the sign and maintain generality by using both Lax eigenfunctions $f_{+}(x, t, \lambda)$ and $f_{-}(x, t, \lambda)$. Thus, the particular solutions of the linear equation (2) for $\kappa=1 / 2$ that we will consider below will be denoted by $h_{ \pm}(x, t, \lambda)$, given by

$h_{ \pm}(x, t, \lambda):=\partial_{x} g_{ \pm}(x, t, \lambda), \quad$ where $\quad g_{ \pm}(x, t, \lambda):=f_{ \pm}(x, t, \lambda) \exp \left(\frac{i}{2}\left(\lambda x+\lambda^{3} t\right)\right)$.

From the Schrödinger equation (6) for $f_{ \pm}(x, t, \lambda)$, it follows that $g_{ \pm}(x, t, \lambda)$ satisfies the ODE

$$
-i \partial_{x}^{2} g_{ \pm}-i \frac{A}{6} g_{ \pm}=\lambda \partial_{x} g_{ \pm} .
$$

This ODE plays an important role in suggesting the completeness relation for the solutions $h_{ \pm}(x, t, \lambda)$.

3. The completeness relation for $\kappa=1 / 2$. Having in hand a large family of exact solutions of the linear equation (2) for $\kappa=1 / 2$ is certainly useful, but we may then ask whether there are enough of these solutions to construct the general solution of the initial value problem by superposition. A completeness relation is a formula that gives the expansion of arbitrary initial data in terms of such a collection of functions. In this section, we will establish the completeness relation for the exact solutions $h_{ \pm}(x, t, \lambda)$ obtained in section 2 .

The form of the completeness relation is suggested by a similar argument to that used by Sachs [S83] in his investigation of the completeness of squared eigenfunction solutions to the linearized $\mathrm{KdV}$ equation. The idea is that ideally we would like to have a differential eigenvalue problem in standard form satisfied by the functions $h_{ \pm}(x, t, \lambda)$ :

$$
L(t) h_{ \pm}(x, t, \lambda)=\lambda h_{ \pm}(x, t, \lambda)
$$

where $\lambda$ is the eigenvalue and $L(t)$ is some second-order linear differential operator in $x$. Then, using the two explicit solutions $h_{ \pm}(x, t, \lambda)$ of this problem, we could solve the inhomogeneous problem

$$
L(t) \psi-\lambda \psi=\phi
$$

by variation of parameters, i.e., by writing $\psi$ as a linear combination of $h_{ \pm}(x, t, \lambda)$ with nonconstant coefficients, and substituting into (18). For a fixed function $\phi(x)$, this determines $\psi(x, t, \lambda)$, and we have thus constructed the resolvent of the operator $L(t)$,

$$
\psi(x, t, \lambda)=(L(t)-\lambda \mathbb{I})^{-1} \phi(x) .
$$

If the spectrum of $L(t)$ is contained in a bounded region of the complex plane (and also under some milder conditions), then the Dunford-Taylor integral of the resolvent 
on a positively oriented contour enclosing the spectrum yields the identity operator

$$
-\frac{1}{2 \pi i} \oint \psi(x, t, \lambda) d \lambda=-\frac{1}{2 \pi i} \oint(L(t)-\lambda \mathbb{I})^{-1} \phi(x) d \lambda=\phi(x) .
$$

However, we do not have a second-order eigenvalue problem for $h_{ \pm}(x, t, \lambda)$. Instead we have the second-order equation (16) for $g_{ \pm}(x, t, \lambda)$. However, we make the guess that a similar procedure will apply here. Namely, for appropriate side conditions (see below) we solve the inhomogeneous equation

$$
-i \partial_{x}^{2} \psi-i \frac{A}{6} \psi-\lambda \partial_{x} \psi=\phi
$$

for $\psi(x, t, \lambda)$ using variation of parameters with the two functions $g_{ \pm}(x, t, \lambda)$ solving the homogeneous equation (16), and then we differentiate the resulting formula with respect to $x$. Formally speaking only, we have thus constructed the resolvent of the "operator"

$$
L(t)=-i \partial_{x}-i \frac{A}{6} \partial_{x}^{-1}
$$

The obstruction to rigor here is that $\partial_{x}^{-1}$ is not well defined. Nonetheless, we are guided to hypothesize that

$$
-\frac{1}{2 \pi i} \oint \partial_{x} \psi(x, t, \lambda) d \lambda=\phi(x)
$$

for an appropriate contour of integration. This formula turns out to be correct, although a direct proof must be supplied. The proof we use follows Miller and Akhmediev [MA98].

3.1. Solving the inhomogeneous problem. We express the solution of the inhomogeneous problem in the form

$$
\psi(x, t, \lambda)=C_{+}(x, t, \lambda) g_{+}(x, t, \lambda)+C_{-}(x, t, \lambda) g_{-}(x, t, \lambda),
$$

subject to the usual "reduction of order" condition

$$
\partial_{x} C_{+}(x, t, \lambda) \cdot g_{+}(x, t, \lambda)+\partial_{x} C_{-}(x, t, \lambda) \cdot g_{-}(x, t, \lambda)=0 .
$$

Substituting (24) into the equation for $\psi$, and using (25), one finds

$$
\partial_{x} C_{+}(x, t, \lambda)=-i \frac{\phi(x) g_{-}(x, t, \lambda)}{W\left(g_{+}, g_{-}\right)} \quad \text { and } \quad \partial_{x} C_{-}(x, t, \lambda)=i \frac{\phi(x) g_{+}(x, t, \lambda)}{W\left(g_{+}, g_{-}\right)}
$$

where $W\left(g_{+}, g_{-}\right):=g_{+} \partial_{x} g_{-}-g_{-} \partial_{x} g_{+}$is the Wronskian.

From the differential equation (16) satisfied by $g_{ \pm}(x, t, \lambda)$, it follows that

$$
\partial_{x} W\left(g_{+}, g_{-}\right)=i \lambda W\left(g_{+}, g_{-}\right) .
$$

Using the large $|x|$ asymptotics of $f_{ \pm}(x, t, \lambda)$ obtained in section 2, one then solves (27) uniquely and finds that

$$
W\left(g_{+}, g_{-}\right)=i \lambda^{1-2 N} \exp \left(i\left(\lambda x+\lambda^{3} t\right)\right) \prod_{n=1}^{N}\left(\lambda^{2}+4 \eta_{n}^{2}\right) .
$$


In solving the inhomogeneous equation $(21)$ for $\psi$, we really should impose appropriate side conditions. Here, the side conditions we use are not related to boundary conditions in $x$ as much as to analyticity conditions in $\lambda$. It is easy to check that for each $x_{0, U}$, the function $\psi_{U}(x, t, \lambda)$ defined by

$$
\begin{aligned}
\psi_{U}(x, t, \lambda)= & -\int_{x_{0}, U}^{x} \frac{g_{-}(z, t, \lambda) \exp \left(-i\left(\lambda z+\lambda^{3} t\right)\right) \phi(z)}{\lambda^{1-2 N} \prod_{n=1}^{N}\left(\lambda^{2}+4 \eta_{n}^{2}\right)} d z \cdot g_{+}(x, t, \lambda) \\
& +\int_{-\infty}^{x} \frac{g_{+}(z, t, \lambda) \exp \left(-i\left(\lambda z+\lambda^{3} t\right)\right) \phi(z)}{\lambda^{1-2 N} \prod_{n=1}^{N}\left(\lambda^{2}+4 \eta_{n}^{2}\right)} d z \cdot g_{-}(x, t, \lambda)
\end{aligned}
$$

is a solution analytic in $\lambda$ for $\Im(\lambda)>0$ and $|\lambda|$ sufficiently large. Similarly, $\psi_{L}(x, t, \lambda)$ defined for each $x_{0, L}$ by

$$
\begin{aligned}
\psi_{L}(x, t, \lambda)= & -\int_{x_{0, L}}^{x} \frac{g_{-}(z, t, \lambda) \exp \left(-i\left(\lambda z+\lambda^{3} t\right)\right) \phi(z)}{\lambda^{1-2 N} \prod_{n=1}^{N}\left(\lambda^{2}+4 \eta_{n}^{2}\right)} d z \cdot g_{+}(x, t, \lambda) \\
& -\int_{x}^{\infty} \frac{g_{+}(z, t, \lambda) \exp \left(-i\left(\lambda z+\lambda^{3} t\right)\right) \phi(z)}{\lambda^{1-2 N} \prod_{n=1}^{N}\left(\lambda^{2}+4 \eta_{n}^{2}\right)} d z \cdot g_{-}(x, t, \lambda)
\end{aligned}
$$

is a solution analytic for $\Im(\lambda)<0$ and $|\lambda|$ sufficiently large. The qualification of $|\lambda|$ being sufficiently large is necessary because the expressions have poles at the soliton eigenvalues in the respective half-planes where the two functions $g_{ \pm}(x, t, \lambda)$ become proportional. However, these are the only finite singularities, and both solutions $\psi_{U}(x, t, \lambda)$ and $\psi_{L}(x, t, \lambda)$ are meromorphic in the whole of their respective open halfplanes.

The arbitrariness of the parameters $x_{0, U}$ and $x_{0, L}$ would seem to be a problem; however, it will turn out that these terms contribute nothing to the Dunford-Taylor integral that we will prove gives the required completeness relation.

3.2. Integrating the resolvent. Here we show that the guess we made is indeed correct.

THEOREM 3.1. Let $\phi(x)$ be an absolutely continuous function in $L^{1}(\mathbb{R})$. Let $x_{0, U}$ and $x_{0, L}$ be constants, and let $t \in \mathbb{R}$ be fixed. Then,

$$
\phi(x)=-\frac{1}{2 \pi i} \lim _{R \rightarrow \infty}\left[\int_{C_{U}} \partial_{x} \psi_{U}(x, t, \lambda) d \lambda+\int_{C_{L}} \partial_{x} \psi_{L}(x, t, \lambda) d \lambda\right]
$$

where $C_{U}$ is the positively oriented half-circle from $R$ to $-R$ in the upper half-plane and $C_{L}$ is the positively oriented half-circle from $-R$ to $R$ in the lower half-plane.

Proof. First, we show that the terms depending on the arbitrary parameters $x_{0, U}$ and $x_{0, L}$ converge to zero as $R \rightarrow \infty$. This will justify calling the function $\psi_{U}$ or $\psi_{L}$ 
a "resolvent" even though the inverse is not unique. Consider the integral

$$
\begin{aligned}
J_{U} & :=\int_{C_{U}} \partial_{x}\left[-\int_{x_{0, U}}^{x} \frac{g_{-}(z, t, \lambda) \exp \left(-i\left(\lambda z+\lambda^{3} t\right)\right) \phi(z)}{\lambda^{1-2 N} \prod_{n=1}^{N}\left(\lambda^{2}+4 \eta_{n}^{2}\right)} d z \cdot g_{+}(x, t, \lambda)\right] d \lambda \\
& =-\int_{C_{U}} \frac{\lambda^{2 N} h_{+}(x, t, \lambda)}{\lambda \prod_{n=1}^{N}\left(\lambda^{2}+4 \eta_{n}^{2}\right)} \int_{x_{0, U}}^{x} g_{-}(z, t, \lambda) \exp \left(-i\left(\lambda z+\lambda^{3} t\right)\right) \phi(z) d z d \lambda
\end{aligned}
$$

where we have used the relation (25). Recall that

$$
\begin{aligned}
h_{+}(x, t, \lambda) & =\sum_{n=1}^{N} \lambda^{-n} \partial_{x} f_{N-n}(x, t), \\
g_{-}(z, t, \lambda) \exp \left(-i\left(\lambda z+\lambda^{3} t\right)\right) & =1+\sum_{n=1}^{N}(-\lambda)^{-n} f_{N-n}(z, t) .
\end{aligned}
$$

Therefore, for all $\lambda$ with $|\lambda|=R>1$,

$$
\left|h_{+}(x, t, \lambda)\right| \leq \frac{1}{R} \sum_{n=1}^{N}\left|\partial_{x} f_{N-n}(x, t)\right|
$$

and

$$
\sup _{z \in \mathbb{R}}\left|g_{-}(z, t, \lambda) \exp \left(-i\left(\lambda z+\lambda^{3} t\right)\right)\right| \leq 1+\sup _{z \in \mathbb{R}} \sum_{n=1}^{N}\left|f_{N-n}(z, t)\right| .
$$

This latter relation assumes the uniform boundedness of the functions $f_{k}(z, t)$ in $z$. Finally, it is clear that for $|\lambda|=R>\sup _{n} 2 \eta_{n}$

$$
\left|\lambda^{1-2 N} \prod_{n=1}^{N}\left(\lambda^{2}+4 \eta_{n}^{2}\right)\right| \geq R \prod_{n=1}^{N}\left(1-\frac{4 \eta_{n}^{2}}{R^{2}}\right) .
$$

It follows that for all $\lambda$ with $|\lambda|=R$ sufficiently large,

$$
\left|J_{U}\right| \leq \frac{K(x, t)}{R}\|\phi\|_{1}
$$

where

$$
K(x, t)=\pi \prod_{n=1}^{N}\left(1-\frac{4 \eta_{n}^{2}}{R^{2}}\right)^{-1} \cdot\left(\sum_{n=1}^{N}\left|\partial_{x} f_{N-n}(x, t)\right|\right) \cdot\left(1+\sup _{z \in \mathbb{R}} \sum_{n=1}^{N}\left|f_{N-n}(z, t)\right|\right) .
$$

The bound (37) clearly vanishes as $R \rightarrow \infty$. A nearly identical argument shows that the integral 
$(39)$

$$
J_{L}:=\int_{C_{L}} \partial_{x}\left[-\int_{x_{0, L}}^{x} \frac{g_{-}(z, t, \lambda) \exp \left(-i\left(\lambda z+\lambda^{3} t\right)\right) \phi(z)}{\lambda^{1-2 N} \prod_{n=1}^{N}\left(\lambda^{2}+4 \eta_{n}^{2}\right)} d z \cdot g_{+}(x, t, \lambda)\right] d \lambda
$$

satisfies the same bound (37) as $J_{U}$.

Now we consider integrating the second terms of $\partial_{x} \psi_{U}(x, t, \lambda)$ and $\partial_{x} \psi_{L}(x, t, \lambda)$, respectively. For brevity, define

$$
Y(x, z, t, \lambda):=\frac{g_{+}(z, t, \lambda) \exp \left(-i\left(\lambda z+\lambda^{3} t\right)\right) h_{-}(x, t, \lambda)}{\lambda^{1-2 N} \prod_{n=1}^{N}\left(\lambda^{2}+4 \eta_{n}^{2}\right)} .
$$

Note that this can be written as

$$
\begin{aligned}
Y(x, z, t, \lambda)= & \exp (i \lambda(x-z)) \frac{\left(1+\sum_{n=1}^{N} \frac{f_{N-n}(z, t)}{\lambda^{n}}\right)}{\lambda \prod_{n=1}^{N}\left(1+\frac{4 \eta_{n}^{2}}{\lambda^{2}}\right)} \\
& \times\left(i \lambda\left(1+\sum_{n=1}^{N} \frac{f_{N-n}(x, t)}{(-\lambda)^{n}}\right)+\sum_{n=1}^{N} \frac{\partial_{x} f_{N-n}(x, t)}{(-\lambda)^{n}}\right),
\end{aligned}
$$

and therefore,

$$
Y(x, z, t, \lambda)=i \exp (i \lambda(x-z))(1+\Delta(x, z, t, \lambda)),
$$

where $\Delta(x, z, t, \lambda)=O\left(\lambda^{-1}\right)$ uniformly in $x$ and $z$ for fixed $t$. It also follows from additional cancellation that for $z=x, \Delta(x, x, t, \lambda)=O\left(\lambda^{-2}\right)$ uniformly in $x$. Finally, derivatives of $\Delta$ are controlled as well: $\partial_{z} \Delta(x, z, t, \lambda)=O\left(\lambda^{-1}\right)$ uniformly. The integral we need to compute for the contribution of $\partial_{x} \psi_{U}(x, t, \lambda)$ is

$$
\begin{aligned}
\int_{C_{U}} \int_{-\infty}^{x} Y(x, z, t, \lambda) \phi(z) d z d \lambda= & i \int_{C_{U}} \int_{-\infty}^{x} \exp (i \lambda(x-z)) \phi(z) d z d \lambda \\
& +i \int_{C_{U}} \int_{-\infty}^{x} \Delta(x, z, t, \lambda) \exp (i \lambda(x-z)) \phi(z) d z d \lambda .
\end{aligned}
$$

Note that since the integrand is analytic in the upper half-plane, the first term can be written as

(44) $i \int_{C_{U}} \int_{-\infty}^{x} \exp (i \lambda(x-z)) \phi(z) d z d \lambda=-i \int_{-R}^{R} \int_{-\infty}^{x} \exp (i \lambda(x-z)) \phi(z) d z d \lambda$.

In order to control the error term, it is necessary to integrate by parts once:

$$
\begin{gathered}
i \int_{C_{U}} \int_{-\infty}^{x} \Delta(x, z, t, \lambda) \exp (i \lambda(x-z)) \phi(z) d z d \lambda=-\phi(x) \int_{C_{U}} \frac{\Delta(x, x, t, \lambda)}{\lambda} d \lambda \\
\quad+\int_{C_{U}} \int_{-\infty}^{x} \frac{\exp (i \lambda(x-z))}{\lambda} \partial_{z}(\Delta(x, z, t, \lambda) \phi(z)) d z d \lambda
\end{gathered}
$$


The boundary term at $z=-\infty$ vanishes because $\Delta(x, z, t, \lambda)$ is bounded there, $\phi$ is continuous and integrable, and $\exp (i \lambda(x-z))$ is exponentially small for $\Im(\lambda)>0$. Since the exponential is bounded in magnitude by unity for $\Im(\lambda)>0$ and the contour is of length $\pi R$, the above estimates of $\Delta$ imply that there exist $K_{0}(x, t), K_{1}(x, t)$, and $K_{2}(x, t)$ all positive, such that

$$
\begin{aligned}
& \left|\int_{C_{U}} \int_{-\infty}^{x} \Delta(x, z, t, \lambda) \exp (i \lambda(x-z)) \phi(z) d z d \lambda\right| \\
& \quad=\frac{K_{0}(x, t)}{R^{2}}|\phi(x)|+\frac{K_{1}(x, t)}{R}\|\phi\|_{1}+\frac{K_{2}(x, t)}{R}\left\|\phi^{\prime}\right\|_{1} .
\end{aligned}
$$

This proves that

$$
\lim _{R \rightarrow \infty} \int_{C_{U}} \int_{-\infty}^{x} Y(x, z, t, \lambda) \phi(z) d z d \lambda=-i \lim _{R \rightarrow \infty} \int_{-R}^{R} \int_{-\infty}^{x} \exp (i \lambda(x-z)) \phi(z) d z d \lambda .
$$

Similar arguments applied to the contribution of $\partial_{x} \psi_{L}(x, t, \lambda)$ show that

$$
-\lim _{R \rightarrow \infty} \int_{C_{L}} \int_{x}^{\infty} Y(x, z, t, \lambda) \phi(z) d z d \lambda=-i \lim _{R \rightarrow \infty} \int_{-R}^{R} \int_{x}^{\infty} \exp (i \lambda(x-z)) \phi(z) d z d \lambda,
$$

and therefore,

$$
\begin{array}{r}
-\frac{1}{2 \pi i} \lim _{R \rightarrow \infty}\left[\int_{C_{U}} \partial_{x} \psi_{U}(x, t, \lambda) d \lambda+\int_{C_{L}} \partial_{x} \psi_{L}(x, t, \lambda) d \lambda\right] \\
=\frac{1}{2 \pi} \lim _{R \rightarrow \infty} \int_{-R}^{R} \int_{-\infty}^{\infty} \exp (i \lambda(x-z)) \phi(z) d z d \lambda=\phi(x)
\end{array}
$$

with the last equality following from Fourier inversion. This establishes (31) and the theorem.

As it stands, the completeness relation given in Theorem 3.1 is not really an expansion of $\phi(x)$ in terms of the functions $h_{-}(x, t, \lambda)$ because the expansion coefficients themselves depend on $x$. This is easily remedied by casting the right-hand side of the completeness relation into a more useful form. We do this now.

THEOREM 3.2. Let $\phi(x)$ be an absolutely continuous function in $L^{1}(\mathbb{R})$. Let $t \in \mathbb{R}$ be fixed, and choose any $w \in \mathbb{R} \cup\{-\infty,+\infty\}$. Define the mode function

$$
H(x, t, \lambda):=\lambda^{N} h_{-}(x, t, \lambda),
$$

which is an entire function of $\lambda$, and the amplitudes

$$
\begin{aligned}
b^{+}(t, \lambda) & :=\int_{w}^{\infty} \frac{\lambda^{N} g_{+}(z, t, \lambda) \exp \left(-i\left(\lambda z+\lambda^{3} t\right)\right)}{\lambda \prod_{n=1}^{N}\left(\lambda^{2}+4 \eta_{n}^{2}\right)} \phi(z) d z, \\
b^{-}(t, \lambda) & :=\int_{-\infty}^{w} \frac{\lambda^{N} g_{+}(z, t, \lambda) \exp \left(-i\left(\lambda z+\lambda^{3} t\right)\right)}{\lambda \prod_{n=1}^{N}\left(\lambda^{2}+4 \eta_{n}^{2}\right)} \phi(z) d z,
\end{aligned}
$$


and set $b(t, \lambda):=b^{+}(t, \lambda)+b^{-}(t, \lambda)$. The amplitudes have simple poles at $\lambda=0$ and $\lambda= \pm 2 i \eta_{n}$ for $n=1, \ldots, N$. Finally, set

$$
b_{0}(t):=\frac{1}{2} \operatorname{Res}_{\lambda=0}\left(b^{+}(t, \lambda)-b^{-}(t, \lambda)\right) \quad \text { and } \quad b_{n}^{ \pm}(t):=\mp \operatorname{Res}_{\lambda= \pm 2 i \eta_{n}} b^{\mp}(t, \lambda) .
$$

Then we have the expansion

$$
\begin{aligned}
\phi(x)= & \lim _{R \rightarrow \infty} \frac{1}{2 \pi i} \mathrm{P} . \mathrm{V} \cdot \int_{-R}^{R} b(t, \lambda) H(x, t, \lambda) d \lambda \\
& +b_{0}(t) H(x, t, 0)+\sum_{n=1}^{N}\left[b_{n}^{-}(t) H\left(x, t,-2 i \eta_{n}\right)+b_{n}^{+}(t) H\left(x, t, 2 i \eta_{n}\right)\right] .
\end{aligned}
$$

Remark 1. Since $w$ is now fixed and not a function of $x$, this expansion (53) is a true completeness relation, expressing an arbitrary given function $\phi(x)$ as a sum of known functions $H(x, t, \lambda)$. From the exact formulas (50), (15), and (8), it is clear that the part of the expansion (53) represented by the singular integral is Fourierlike, with the corresponding components of the solution, $H(x, t, \lambda)$ for $\lambda \in \mathbb{R}$ being bounded oscillatory functions tending to complex exponentials for large $x$. On the other hand, the discrete contributions to the solution represent bound states. The $2 N+1$ bound state terms in (53) are not linearly independent. From the fact that at the eigenvalues $\pm 2 i \eta_{n}$ the functions $g_{-}(x, t, \lambda)$ are all linear combinations of the same $N$ functions $f_{0}(x, t), \ldots, f_{N-1}(x, t)$, it is clear that only $N$ of the bound states are linearly independent. These facts are easiest to see when one takes $w$ to $\infty$ or $-\infty$. Then, half of the contributions from the eigenvalues disappear, and it remains only to express the bound state at zero, $H(x, t, 0)$, in terms of $H\left(x, t, \pm 2 i \eta_{n}\right)$. This can be done directly. From the exact formulas (50), (15), and (8), we see that

$$
H(x, t, 0)=(-1)^{N} \partial_{x} f_{0}(x, t),
$$

and making use of the relations (9) satisfied by $f_{+}$at the eigenvalues,

$$
H\left(x, t, 2 i \eta_{n}\right)=(-1)^{n+1} \exp \left(-2 \eta_{n} \alpha_{n}\right) \sum_{p=0}^{N-1}\left(2 i \eta_{n}\right)^{p} \partial_{x} f_{p}(x, t) .
$$

Expressing $H(x, t, 0)$ in terms of $H\left(x, t, 2 i \eta_{n}\right)$ is therefore a polynomial interpolation problem. Introduce the polynomial

$$
P(\lambda)=\sum_{p=0}^{N-1} \partial_{x} f_{p}(x, t) \lambda^{p} .
$$

Given isolated values of this polynomial

$$
P\left(2 i \eta_{n}\right)=(-1)^{n+1} \exp \left(2 \eta_{n} \alpha_{n}\right) H\left(x, t, 2 i \eta_{n}\right) \quad \text { for } \quad n=1, \ldots, N,
$$

we are to find $P(0)$ and thus $H(x, t, 0)=(-1)^{N} \partial_{x} f_{0}(x, t)=(-1)^{N} P(0)$. Expressing $P(\lambda)$ explicitly in terms of Lagrange polynomials gives

$$
P(\lambda)=\sum_{n=1}^{N}(-1)^{n+1} \exp \left(2 \eta_{n} \alpha_{n}\right) H\left(x, t, 2 i \eta_{n}\right) \prod_{k \neq n} \frac{\lambda-2 i \eta_{k}}{2 i \eta_{n}-2 i \eta_{k}},
$$


and therefore

$$
H(x, t, 0)=\sum_{n=1}^{N}\left[(-1)^{n} \exp \left(2 \eta_{n} \alpha_{n}\right) \prod_{k \neq n} \frac{\eta_{k}}{\eta_{n}-\eta_{k}}\right] H\left(x, t, 2 i \eta_{n}\right) .
$$

There is, indeed, a similar expression for $H(x, t, 0)$ in terms of $H\left(x, t,-2 i \eta_{n}\right)$.

Remark 2. An important distinction between the completeness relation stated in Theorem 3.2 and that found by Sachs [S83] for derivatives of squared Schrödinger eigenfunctions is in the nature of the singularity at $\lambda=0$. Sachs shows that in the expansion of $\phi$ in terms of derivatives of squared eigenfunctions, there is an apparent singularity at $\lambda=0$ that is in fact removable. On the other hand, the integral in Theorem 3.2 is essentially singular and the residue contribution of $b_{0}(t)$ is nonzero.

Proof of Theorem 3.2. We first establish that in the formulas (29) for $\partial_{x} \psi_{U}(x, t, \lambda)$ and (30) for $\partial_{x} \psi_{L}(x, t, \lambda)$ we may replace $x$ in the limits of integration by any other value without changing the result of the theorem. That is, we will now show that the integral

$$
\int_{C_{U}} \int_{-\infty}^{w} Y(x, z, t, \lambda) \phi(z) d z d \lambda-\int_{C_{L}} \int_{w}^{\infty} Y(x, z, t, \lambda) \phi(z) d z d \lambda,
$$

which we have already seen converges as $R$ tends to infinity to $-2 \pi i \phi(x)$ in the case that $w=x$, is in fact independent of $w$. Holding $R$ fixed and differentiating with respect to $w$, we must show that for sufficiently large $R$,

$$
\phi(w) \oint_{|\lambda|=R} Y(x, w, t, \lambda) d \lambda \equiv 0
$$

identically in $x, w$, and $t$. Being as the integrand is meromorphic in the finite $\lambda$ plane, we can evaluate the integral by residues. There are simple poles at $\lambda=0$ and $\lambda= \pm 2 i \eta_{n}$ for $n=1, \ldots, N$. Using the linear relations (9) satisfied by $f_{ \pm}$at the eigenvalues $\lambda=2 i \eta_{n}$, we find

$$
\begin{aligned}
\operatorname{Res}_{\lambda=2 i \eta_{k}} Y(x, w, t, \lambda) & =\sum_{p=0}^{N-1} \frac{\left(2 i \eta_{k}\right)^{N+p-1}}{D_{k}} \partial_{x} f_{p}(x, t) \\
& +\sum_{p, q=0}^{N-1}(-1)^{N-q} \frac{\left(2 i \eta_{k}\right)^{p+q-1}}{D_{k}} f_{q}(w, t) \partial_{x} f_{p}(x, t), \\
\operatorname{Res}_{\substack{\lambda=-2 i \eta_{k} \\
Y}} Y(x, w, t, \lambda)= & \sum_{p=0}^{N-1}(-1)^{N-p} \frac{\left(2 i \eta_{k}\right)^{N+p-1}}{D_{k}} \partial_{x} f_{p}(x, t) \\
& +\sum_{p, q=0}^{N-1}(-1)^{N-p} \frac{\left(2 i \eta_{k}\right)^{p+q-1}}{D_{k}} f_{q}(w, t) \partial_{x} f_{p}(x, t),
\end{aligned}
$$

where

$$
D_{k}:=\prod_{n \neq k}\left(2 i \eta_{k}-2 i \eta_{n}\right) \prod_{n=1}^{N}\left(2 i \eta_{k}+2 i \eta_{n}\right)
$$


Similarly, for the residue at zero,

$$
\operatorname{Res}_{\lambda=0} Y(x, w, t, \lambda)=(-1)^{N} \frac{f_{0}(w, t) \partial_{x} f_{0}(x, t)}{\prod_{n=1}^{N} 4 \eta_{n}^{2}} .
$$

Adding all the residues and collecting coefficients of the terms $\partial_{x} f_{p}(x, t)$ and $f_{q}(w, t)$ $\partial_{x} f_{p}(x, t)$, we find that the sum of the residues will be zero if

$$
I_{p}:=\sum_{k=1}^{N} \frac{\left(2 i \eta_{k}\right)^{p}}{D_{k}}=0
$$

for all odd $p=1,3,5, \ldots, 2 N-3$, and if

$$
\frac{1}{\prod_{n=1}^{N} 4 \eta_{n}^{2}}+2 \sum_{k=1}^{N} \frac{1}{2 i \eta_{k} D_{k}}=0 .
$$

These expressions are themselves sums of residues of meromorphic differentials. Thus, by inspection, one finds that for $p=1,3,5, \ldots, 2 N-3$,

$$
I_{p}=\frac{1}{2 \pi i} \oint_{C} \frac{\lambda^{p} d \lambda}{\prod_{n=1}^{N}\left(\lambda^{2}+4 \eta_{n}^{2}\right)},
$$

where $C$ is any simple counterclockwise oriented contour that encircles the points $\lambda=2 i \eta_{n}$ for $n=1, \ldots, N$ (but without enclosing the conjugate eigenvalues or $\lambda=0$ ). With $p$ bounded by $2 N-3$, the path of integration can be blown out to infinity in the upper half-plane and then brought down to the real axis so that

$$
I_{p}=\frac{1}{2 \pi i} \int_{-\infty}^{\infty} \frac{\lambda^{p} d \lambda}{\prod_{n=1}^{N}\left(\lambda^{2}+4 \eta_{n}^{2}\right)}=0
$$

with the last equality following from the oddness of the integrand for odd $p$. Finally, consider the integral $I_{-1}$ defined by

$$
I_{-1}:=\frac{1}{2 \pi i} \oint_{C} \frac{d \lambda}{\lambda \prod_{n=1}^{N}\left(\lambda^{2}+4 \eta_{n}^{2}\right)} .
$$

Evaluating the residues inside $C$, we find

$$
I_{-1}=\sum_{k=1}^{N} \frac{1}{2 i \eta_{k} \prod_{n \neq k}\left(2 i \eta_{k}-2 i \eta_{n}\right) \prod_{n=1}^{N}\left(2 i \eta_{k}+2 i \eta_{n}\right)}
$$


On the other hand, we can again blow the contour $C$ out to infinity in the upper half-plane and bring it down to the real axis. This time, there is a singularity at $\lambda=0$, so the Plemelj formula must be used. We find

$$
I_{-1}=-\frac{1}{2} \cdot \frac{1}{\prod_{n=1}^{N} 4 \eta_{n}^{2}}+\frac{1}{2 \pi i} \mathrm{P} . \mathrm{V} \cdot \int_{-\infty}^{\infty} \frac{d \lambda}{\lambda \prod_{n=1}^{N}\left(\lambda^{2}+4 \eta_{n}^{2}\right)}
$$

Once again, by oddness, the principal value integral vanishes identically, and then combining this result with the previous expression, we obtain the required vanishing.

This shows that for any $w$,

$\phi(x)=-\frac{1}{2 \pi i} \lim _{R \rightarrow \infty}\left[\int_{C_{U}} \int_{-\infty}^{w} Y(x, z, t, \lambda) \phi(z) d z d \lambda-\int_{C_{L}} \int_{w}^{\infty} Y(x, z, t, \lambda) \phi(z) d z d \lambda\right]$.

Establishing (53) and therefore the theorem now amounts to using the residue theorem once again to deform the integration paths $C_{U}$ and $C_{L}$ in (72) to the real axis. One finds discrete contributions at the poles $\lambda= \pm 2 i \eta_{n}$, and then applying the Plemelj formula to contract the contour to the real axis in the neighborhood of $\lambda=0$ gives a discrete contribution proportional to $H(x, t, 0)$ and the principal value regularization of the singular integral over the continuous spectrum.

4. Solution of the initial value problem for $\kappa=1 / 2$. It is easy to see that when $A(x, t)$ is an $N$-soliton solution of $\mathrm{KdV}(1)$, one can use the completeness relation to solve the initial value problem

$$
\partial_{t} B+\partial_{x}\left[\frac{1}{2} A B+\partial_{x}^{2} B\right]=0, \quad B(x, 0)=\phi(x) .
$$

Setting $t=0$, and picking a convenient value of $w$, say, $w=+\infty$, one computes the amplitudes (51) and discrete coefficients (52). Then, because the function $H(x, t, \lambda)$ satisfies (2) for $\kappa=1 / 2$ and for each complex $\lambda$, the expression

$$
\begin{aligned}
B(x, t):= & \lim _{R \rightarrow \infty} \frac{1}{2 \pi i} \mathrm{P} . \mathrm{V} \cdot \int_{-R}^{R} b(0, \lambda) H(x, t, \lambda) d \lambda \\
& +b_{0}(0) H(x, t, 0)+\sum_{n=1}^{N}\left[b_{n}^{-}(0) H\left(x, t,-2 i \eta_{n}\right)+b_{n}^{+}(0) H\left(x, t, 2 i \eta_{n}\right)\right],
\end{aligned}
$$

provides the solution of the initial value problem (73), generally in the sense of distributions. That is, $B(x, 0)=\phi(x)$ by Theorem 3.2 , and for each test function $\varphi(x, t)$ that is differentiable in $t$ and three times differentiable in $x$ and has compact support in $(x, t) \in \mathbb{R} \times \mathbb{R}_{+}$, one shows by exchanging the order of integration that

$$
\int_{0}^{\infty} \int_{-\infty}^{\infty}\left[\partial_{t} \varphi(x, t)+\frac{1}{2} A(x, t) \partial_{x} \varphi(x, t)+\partial_{x}^{3} \varphi(x, t)\right] B(x, t) d x d t=0 .
$$

The solution will be classical in as much as it is possible to differentiate with respect to $x$ and $t$ under the integral sign in the solution formula (74). This requires additional smoothness and decay assumptions on the initial data $\phi(x)$ that we do not consider here. 
5. Scattering of bound states for $\kappa=\mathbf{1 / 2}$. Of particular interest in applications is the $N$-dimensional (recall that $A(x, t)$ is an $N$-soliton solution of $\mathrm{KdV}$ ) subspace of solutions of (2) for $\kappa=1 / 2$ consisting of bound states. This subspace represents linear waves that are trapped by the solitons of the potential $A(x, t)$. For large $|t|$, these bound state solutions are all confined to the trajectories of the solitons. Therefore, it follows that each bound state $B(x, t)$ has two asymptotic representations:

$$
B(x, t) \sim \sum_{n=1}^{N} \beta_{n}^{ \pm} A_{n}^{ \pm}(x, t), \quad t \rightarrow \pm \infty,
$$

for some constants $\beta_{n}^{ \pm}$depending on $B(x, t)$, where $A_{n}^{ \pm}(x, t)$ are defined by (11). Since there are exactly $N$ linearly independent bound states, it follows that the constants $\beta_{n}^{+}$are completely determined from the constants $\beta_{n}^{-}$. In particular, there exists an invertible $N \times N$ matrix $\mathbf{T}$ with entries depending only on the data specifying the $N$-soliton solution $A(x, t)$, such that

$$
\beta_{j}^{+}=\sum_{k=1}^{N} T_{j k} \beta_{k}^{-} .
$$

The matrix $\mathbf{T}$ is called the bound state scattering matrix. In this section, we compute the scattering matrix explicitly and show that its elements only depend on the soliton eigenvalues $\eta_{1}, \ldots, \eta_{N}$.

If $A(x, t)$ is an $N$-soliton solution of $\mathrm{KdV}(1)$, then a family of solutions to (2) for $\kappa=1 / 2$, parametrized by complex $\lambda$, is given by

$$
h_{+}(x, t, \lambda)=\frac{A(x, t)}{6 i \lambda}+\sum_{n=0}^{N-2} \lambda^{n-N} \partial_{x} f_{n}(x, t) .
$$

We want to analyze these solutions in the limit of large $|t|$, in a frame of reference traveling with constant velocity $c$.

The first step is to see how the coefficients $f_{n}(x, t)$ behave for large $|t|$. Let $\chi=$ $x-c t$ be fixed as $\tau=t$ goes to either $+\infty$ or $-\infty$. Begin by taking $\eta_{m}^{2}<4 c<\eta_{m-1}^{2}$ to see how the coefficients behave in between the solitons. In the limit $\tau \rightarrow+\infty$, the equations (9) imply that

$$
\begin{aligned}
& 1+\sum_{k=0}^{N-1}\left(-2 i \eta_{n}\right)^{k-N} f_{k} \rightarrow 0, \quad n=1, \ldots, m-1, \\
& 1+\sum_{k=0}^{N-1}\left(2 i \eta_{n}\right)^{k-N} f_{k} \rightarrow 0, \quad n=m, \ldots, N .
\end{aligned}
$$

This is an invertible Vandermonde system for the coefficients $f_{k}$, so that as $\tau \rightarrow$ $+\infty$, the $f_{k}$ all become constants, independent of $\chi$ and $\tau$. Thus, $\partial_{x} f_{k}(x, t)$ vanishes between the solitons for all $k$. The analogous result holds as $\tau \rightarrow-\infty$. This shows that the solutions of (2) for $\kappa=1 / 2$ described by the formula (78) are asymptotically confined to the individual frames of reference of the moving solitons in the potential field $A(x, t)$. 
Now set $c=4 \eta_{m}^{2}$ to go into the moving frame of reference of one of the solitons. Taking the limit $\tau \rightarrow+\infty$ yields

$$
\begin{aligned}
1 & +\sum_{k=0}^{N-1}\left(-2 i \eta_{n}\right)^{k-N} f_{k} \rightarrow 0, \quad n=1, \ldots, m-1 \\
1+ & \sum_{k=0}^{N-1}\left(2 i \eta_{n}\right)^{k-N} f_{k} \quad \rightarrow \quad 0, \quad n=m+1, \ldots, N
\end{aligned}
$$

This is a system of $N-1$ equations in $N$ unknowns, so it can be used to asymptotically eliminate $\partial_{\chi} f_{0}$ through $\partial_{\chi} f_{N-2}$ in favor of $\partial_{\chi} f_{N-1}$, which we know is proportional to the $N$-soliton solution of $\mathrm{KdV}, A(x, t)$. Thus, as $\tau \rightarrow+\infty$, with $c=4 \eta_{m}^{2}$,

$$
\partial_{\chi} f_{k}=Q_{m k} \partial_{\chi} f_{N-1}=\frac{1}{6 i} Q_{m k} A
$$

for $k=0, \ldots, N-2$, where the numbers $Q_{m k}$ are the unique solution of the inhomogeneous system of linear algebraic equations

$$
\begin{aligned}
\left(-2 i \eta_{n}\right)^{-1} & +\sum_{k=0}^{N-2}\left(-2 i \eta_{n}\right)^{k-N} Q_{m k}=0, \quad n=1, \ldots, m-1, \\
\left(2 i \eta_{n}\right)^{-1} & +\sum_{k=0}^{N-2}\left(2 i \eta_{n}\right)^{k-N} Q_{m k}=0, \quad n=m+1, \ldots, N .
\end{aligned}
$$

One can similarly show that as $\tau \rightarrow-\infty$, with $c=4 \eta_{m}^{2}$,

$$
\partial_{\chi} f_{k}=Q_{m k}^{*} \partial_{\chi} f_{N-1}=\frac{1}{6 i} Q_{m k}^{*} A
$$

for $k=0, \ldots, N-2$, where the star denotes complex conjugation.

Now consider particular solutions $B_{j}(x, t)$ of $(2)$ for $\kappa=1 / 2$ obtained as linear combinations of $N$ others expressed by the formula (78) evaluated on the $N$ soliton eigenvalues. The formula for $B_{j}(x, t)$ is

$$
B_{j}(x, t)=\sum_{k=1}^{N} F_{j k} h_{+}\left(x, t, 2 i \eta_{k}\right)=\sum_{k=1}^{N} F_{j k}\left[-\frac{A(x, t)}{12 \eta_{k}}+\sum_{n=0}^{N-2}\left(2 i \eta_{k}\right)^{n-N} \partial_{x} f_{n}(x, t)\right]
$$

where $\mathbf{F}=\left\{F_{j k}\right\}$ is a matrix of arbitrary constants. From the asymptotics of $f_{n}(x, t)$, we have as $\tau \rightarrow-\infty$ with $c=4 \eta_{m}^{2}$

$$
B_{j} \rightarrow A \sum_{k=1}^{N} F_{j k} G_{k m}^{-}, \quad \text { where } \quad G_{k m}^{-}:=-\frac{1}{12 \eta_{k}}+\frac{1}{6 i} \sum_{n=0}^{N-2}\left(2 i \eta_{k}\right)^{n-N} Q_{m n}^{*}
$$

So, with the choice that the matrix $\left\{F_{j k}\right\}$ is the inverse of the matrix $\mathbf{G}^{-}=\left\{G_{k m}^{-}\right\}$, the particular solution $B_{j}(x, t)$ of $(2)$ for $\kappa=1 / 2$ will be completely confined as $t \rightarrow-\infty$ to the frame of reference moving with speed $c=4 \eta_{j}^{2}$, where it will be locally indistinguishable from the solution $A(x, t)$ of $\mathrm{KdV}$. Let us now determine how $B_{j}(x, t)$ 
will behave in the various soliton frames as $t \rightarrow+\infty$. Passing to the limit of $\tau \rightarrow+\infty$ in the frame with velocity $c=4 \eta_{m}^{2}$ gives

$$
B_{j} \rightarrow A \sum_{k=1}^{N} F_{j k} G_{k m}^{+}, \quad \text { where } \quad G_{k m}^{+}:=-\frac{1}{12 \eta_{k}}+\frac{1}{6 i} \sum_{n=0}^{N-2}\left(2 i \eta_{k}\right)^{n-N} Q_{m n}
$$

These asymptotics give us a formula for the bound state scattering matrix:

$$
\mathbf{T}:=\left[\left(\mathbf{G}^{-}\right)^{-1} \mathbf{G}^{+}\right]^{T} .
$$

It is clear that the elements of $\mathbf{T}$ depend only on the $N$ soliton eigenvalues $\eta_{1}, \ldots, \eta_{N}$. There is no dependence on the soliton phase variables $\alpha_{1}, \ldots, \alpha_{N}$. Therefore, the asymptotic scattering properties of linear waves in (2) with $\kappa=1 / 2$ are insensitive to phase shifts among the solitons in the potential $A(x, t)$. As a concrete example of the scattering matrix, we compute it explicitly for $N=2$ for arbitrary $\eta_{1}>\eta_{2}>0$ :

$$
\mathbf{T}=\frac{1}{\eta_{1}^{2}-\eta_{2}^{2}}\left[\begin{array}{cc}
\left(\eta_{1}-\eta_{2}\right)^{2} & 2 \eta_{2}\left(\eta_{1}-\eta_{2}\right) \\
2 \eta_{1}\left(\eta_{1}-\eta_{2}\right) & -\left(\eta_{1}-\eta_{2}\right)^{2}
\end{array}\right] .
$$

The fact that $T_{22}$ is negative means that it is possible for the interactions of the solitons in $A(x, t)$ to convert trapped linear waves of elevation into waves of depression, and vice-versa.

6. General values of $\boldsymbol{\kappa}$. We expect that for most values of $\kappa$, the linear waves satisfying (2) will not be permanently trapped by solitons present in the potential $A(x, t)$. This is suggested by considering the simplest case, namely, taking $A(x, t)$ to be the one-soliton solution of $\mathrm{KdV}(1)$. The soliton travels with velocity $c=4 \eta^{2}$ so that $A=-V(\chi)$ with $\chi=x-c t-\alpha$. Corresponding traveling wave solutions $B(\chi)$ of the linear problem that propagate with the same velocity and decay as $\chi \rightarrow \pm \infty$ satisfy

$$
-B^{\prime \prime}(\chi)+\kappa V(\chi) B(\chi)=-c B(\chi) .
$$

Since $c$ is fixed, we can view this as an eigenvalue equation with $\kappa$ as the eigenvalue. We therefore expect that only isolated values of $\kappa$ will admit nontrivial decaying solutions $B(\chi)$. We have already seen that $\kappa=1 / 2$ and $\kappa=1$ are indeed eigenvalues. For $\kappa=1 / 2$ the eigenfunction $B(\chi)$ is an even function of $\chi$, while for $\kappa=1$ the eigenfunction $B(\chi)$ is odd in $\chi$. Since eigenfunctions of (89) must be nondegenerate and therefore have either odd or even parity in $\chi$, there cannot exist a nontrivial bound state eigenfunction of (89) for all $\kappa \in[1 / 2,1]$ because the eigenfunction would have to change parity from one endpoint to the other. Therefore, at least one value of $\kappa \in[1 / 2,1]$ is not an eigenvalue. For such $\kappa$, there is no bound state traveling wave solution of (2) that is trapped in the soliton trajectory.

We can be more precise about this phenomenon. The left-hand side of (89) can also be viewed as a Schrödinger operator $L(\kappa)$ depending on a coupling constant $\kappa$, and the condition for wave trapping by solitons is simply that $-c \in \Sigma_{\mathrm{p}}(L(\kappa))$, where $\Sigma_{\mathrm{p}}$ denotes the point spectrum. The number of discrete eigenvalues is a nondecreasing function of $\kappa>0$, corresponding to the deepening of the potential well. There is an infinite unbounded sequence of cutoff values $\kappa_{n}^{\text {cut }}$ of $\kappa$ at which the number of eigenvalues changes by one, and the new eigenvalue is born from the continuum. Each eigenvalue, once born, is distinct and is a decreasing function of $\kappa$. From these 
arguments, it follows that there exists an infinite unbounded sequence of bifurcation values $\kappa_{n}^{\text {bif }}$ of $\kappa$ at which one eigenvalue crosses the level $E=-c$, and a bound state traveling wave solution of (2) exists.

It is easy to find the bifurcation points because the hyperbolic secant squared potential is so well understood. The potential $\kappa V(\chi)$ is exactly reflectionless for $12 \kappa=n(n+1)$ for $n=1,2,3, \ldots$. The corresponding energy levels are $E_{n, k}=-k^{2} \eta^{2}$ for $k=1, \ldots, n$. Therefore, for $n>1$ in this sequence, there is always one eigenvalue that is exactly equal to $-c=-4 \eta^{2}$. The corresponding eigenstate is always the $(n-1)$ st state and therefore has $n-2$ zeros. It follows that the bifurcation points are $\kappa=\kappa_{n}^{\text {bif }}=(n+1)(n+2) / 12$ for $n=1,2,3, \ldots$

The fact that some linear waves may be permanently trapped by isolated solitons at a bifurcation point $\kappa=\kappa_{n}^{\text {bif }}$ does not necessarily imply that there will be no losses to radiation when solitons in the field $A(x, t)$ interact with one another. Such a lossless interaction might suggest the "integrability" of the linear equation (2). We have indeed seen that this is the case for the first two bifurcation points, $\kappa=\kappa_{1}^{\text {bif }}=1 / 2$ and $\kappa=\kappa_{2}^{\text {bif }}=1$, but it is by no means clear that the trend continues for higher-order bifurcation points. For the rest of this section, we therefore restrict attention to the case $N=1$, that is, we take the nonlinear field $A(x, t)$ to be a one-soliton solution of $\mathrm{KdV}(1)$.

Using (3) and the change of variables $x^{\prime}=\eta\left(x-4 \eta^{2} t-\alpha\right)$ and $t^{\prime}=\eta^{3} t,(2)$ becomes, after dropping primes,

$$
\partial_{t} B+\partial_{x}\left[-4 B+12 \kappa \operatorname{sech}^{2}(x) B+\partial_{x}^{2} B\right]=0 .
$$

This equation is of course solved by separation of variables. We seek separated solutions $B(x, t)=b_{\sigma}(x) \exp (\sigma t)$ and obtain the third-order eigenvalue problem

$$
\left[4 b_{\sigma}(x)-12 \kappa \operatorname{sech}^{2}(x) b_{\sigma}(x)-b_{\sigma}^{\prime \prime}(x)\right]^{\prime}=\sigma b_{\sigma}(x),
$$

where the prime denotes differentiation with respect to $x$. In this context, what we have been calling "trapped linear waves" correspond to bound-state eigenfunctions of (91) with $\sigma=0$. Such solutions have finite mass and energy and are stationary in the moving frame of reference of the soliton $A(x, t)$. As we know, such eigenfunctions with $\sigma=0$ exist only at the bifurcation values of $\kappa=\kappa_{n}^{\text {bif }}$. However, it is clear that for general values of $\kappa$ there are other possibilities. There may be eigenvalues $\sigma$ that are purely imaginary, giving rise to oscillating modes that travel in the soliton frame. More generally, if an eigenvalue has a nonzero real part for some $\kappa$, then there will be a mode that is either amplified or exponentially damped as it propagates with the soliton.

The eigenvalue problem (91) has two simple symmetries. Whenever $b_{\sigma}(x)$ is an eigenfunction with eigenvalue $\sigma$, then $b_{\sigma}(-x)$ is an eigenfunction with eigenvalue $-\sigma$ and $b_{\sigma}(x)^{*}$ is an eigenfunction with eigenvalue $\sigma^{*}$. Therefore, the eigenvalues either come in purely real pairs $(|\sigma|,-|\sigma|)$, purely imaginary pairs $(i|\sigma|,-i|\sigma|)$, or in complex quartets $\left(\sigma,-\sigma, \sigma^{*},-\sigma^{*}\right)$. These symmetries indicate the distinguished role of $\sigma=0$ as a point that if it appears in the spectrum for some $\kappa$ can signal a bifurcation in the number of eigenvalues. This explains our terminology and notation for the values $\kappa=\kappa_{n}^{\text {bif }}$.

Most points on the imaginary $\sigma$ axis correspond to continuous spectrum. This can be seen by the following argument. Let $\kappa$ be fixed. Suppose $\sigma=i \omega$ with $\omega \in \mathbb{R}$. For large $|x|$, the solutions of (91) have the form of linear combinations of $\exp \left(i k_{\omega, j} x\right)$ where $k=k_{\omega, j}$ are the three roots of $k^{3}+4 k-\omega=0$. Exactly one of these roots, 
say, $k=k_{\omega, 0}$, is real, while the other two form a complex-conjugate pair. If we seek a generalized eigenfunction normalized to $\exp \left(i k_{\omega, 0} x\right)$ as $x \rightarrow-\infty$ through a "shooting" method, we have three complex constants to exploit: the coefficient of the decaying mode for large negative $x$, and the coefficients of the decaying mode for large positive $x$ and the finite amplitude contribution for large positive $x$. Matching the values of $b_{i \omega}(x), b_{i \omega}^{\prime}(x)$, and $b_{i \omega}^{\prime \prime}(x)$ at $x=0$ gives three complex equations in three complex unknowns. If this system of equations is solvable at all, one expects it to be solvable for almost all real $\omega$, yielding a generalized eigenfunction. For exceptional values of $\omega$ where there is not a generalized eigenfunction, there will be a genuine bound-state eigenfunction since the spectrum is a closed set.

We have used a numerical Fourier-based collocation (pseudospectral) method to find the discrete eigenvalues of (91) over a range of values of the coupling constant $\kappa$. Essentially this involves approximating the continuous function $b_{\sigma}(x)$ by a periodic discrete series. Then the derivative $\partial_{x}$ can be approximated to exponential accuracy by a derivative matrix $\mathbf{D}$ for which an explicit formula is given in [CHQZ88]. This is then used to construct a discrete approximation to the operator on the left-hand side of (91). Standard techniques can then be used to obtain the eigenvalues and eigenvectors of this matrix. The corresponding eigenfunctions always decay exponentially, but sometimes they decay very slowly for large $x$ of one or the other sign-luckily not both. To obtain accurate results it was necessary in these cases to change the dependent variable by multiplying by an appropriate exponential function of $x$ to enhance the decay on the slowly decaying side without changing decay into growth on the other side. Our results over the range $0<\kappa<5$ are shown in Figure 6.1. The bifurcation values $\kappa_{n}^{\text {bif }}$ appear to be of two different types. If $n$ is odd, then when $\kappa$ increases through the value $\kappa=\kappa_{n}^{\text {bif }}$, a new pair of real eigenvalues is born from the origin $\sigma=0$. As $\kappa$ is further increased, the pair of eigenvalues moves at first away from the imaginary axis and then changes direction and contracts toward the origin. When $\kappa$ increases through the even bifurcation value $\kappa=\kappa_{n+1}^{\text {bif }}$, the pair enters the origin and re-emerges as a complex eigenvalue quartet. Further increasing the value of $\kappa$ causes the quartet of eigenvalues to move through a maximum in the magnitude of the real part and then toward the imaginary axis with the magnitude of the real part decreasing to zero while the magnitude of the imaginary part increases without bound. It does not appear that the quartet of eigenvalues ever disappears into the continuous spectrum, although it comes arbitrarily close as $\kappa$ increases. This scenario is repeated again and again as $\kappa$ increases through each odd bifurcation value. Representative eigenfunctions are plotted in Figure 6.2. Here, one can see that when the eigenvalue $\sigma$ has a nonzero imaginary part, the decay of the eigenfunction can be quite slow on the "downstream" side of the soliton. As remarked above, this effect is compensated for in our numerics by working with a modified eigenfunction.

There are no discrete eigenvalues at all for $\kappa<1 / 2$ (and in particular for $\kappa<0$ ), and for all $\kappa$ satisfying $1 / 2<\kappa<1$ and $\kappa>1$, there is always at least one eigenvalue with a nonzero real part, which corresponds to an exponentially growing eigenfunction and therefore instability. The values $\kappa=1 / 2$ and $\kappa=1$ are distinguished as the only values for which there exist discrete eigenvalues and at the same time all eigenvalues have zero real parts, so the system is neutrally stable. For all other values of $\kappa$, either there are no discrete eigenvalues at all in which case all initial conditions for (2) disperse away algebraically in time, or there are discrete eigenvalues with positive real parts in which case the linear waves are amplified by the soliton in the field $A(x, t)$. 

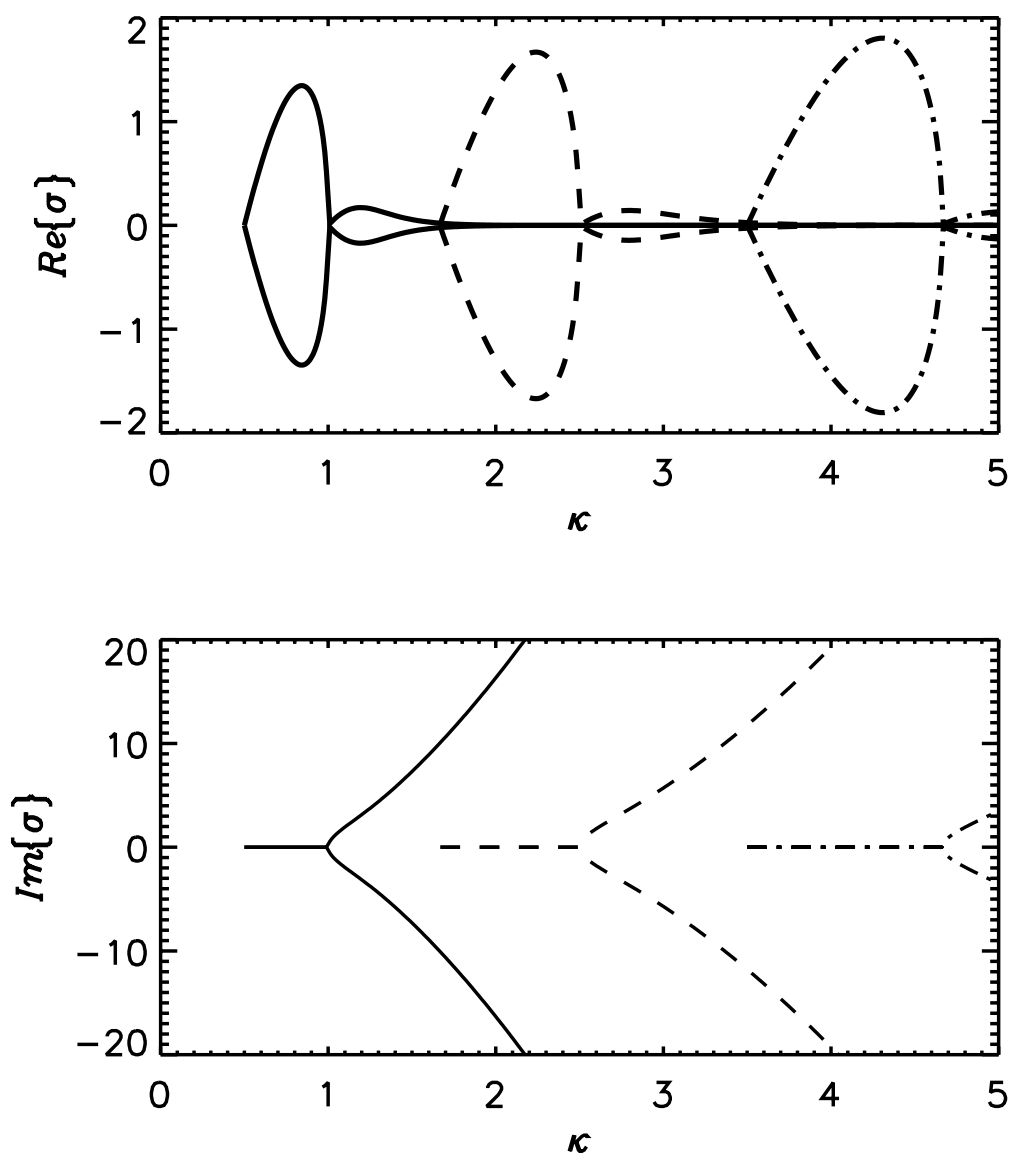

FIG. 6.1. Real (above) and imaginary (below) parts of the discrete eigenvalues $\sigma$ for the eigenvalue problem (91) as a function of the parameter $\kappa$. Different eigenvalue branches are displayed with different styles of lines (solid, dashed, etc.).

7. Conclusion. The coupled system consisting of the KdV equation (1) and the linear equation (2) is integrable for two distinct values of the coupling parameter $\kappa$. The integrable case of $\kappa=1$ has been studied by other authors [GGKM74, S83]. In this paper, we have given new results for the other integrable case, namely, $\kappa=1 / 2$. In particular, we have shown how to construct the general solution of (2) for $\kappa=1 / 2$ when the nonlinear field $A(x, t)$ is an $N$-soliton solution of the KdV equation. This general solution is represented in terms of a number of bound states (equal to the number $N$ of solitons in the field $A(x, t)$ ) and a continuum superposition of radiative states given by a singular integral. With the help of numerical computations, we have placed the integrable cases in context by examining the behavior of the linear equation (2) for general values of $\kappa$, when $A(x, t)$ is a one-soliton solution of $\mathrm{KdV}$. These calculations show that the linear equation (2) behaves as an unstable dynamical system for most positive $\kappa$. The integrable value of $\kappa=1$, for which the equation (2) is the linearized KdV equation, is an isolated stable point, since a small change of either sign in the value of $\kappa$ will lead to the presence of exponentially growing modes. The other integrable value of $\kappa=1 / 2$ represents the boundary between a 

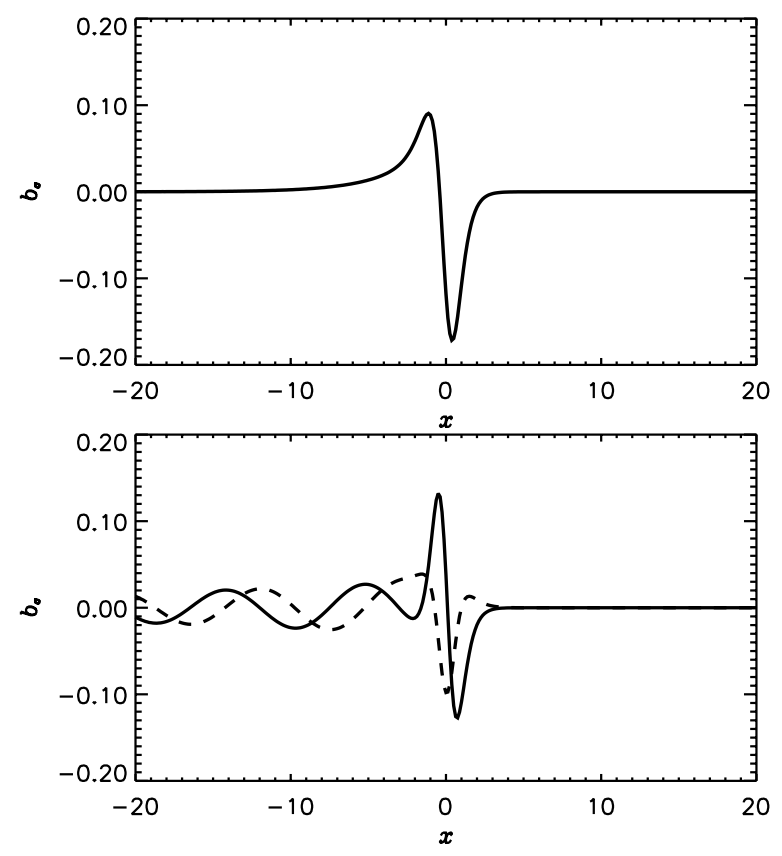

FIG. 6.2. Above: the real-valued eigenfunction corresponding to the eigenvalue $\sigma$ with positive real part for $\kappa=0.85$. Below: the complex-valued eigenfunction corresponding to the eigenvalue $\sigma$ in the first quadrant for $\kappa=1.2$. The solid curve is the real part and the dashed curve is the imaginary part.

stable system without any bound states for $\kappa<1 / 2$ and an exponentially unstable system for $\kappa>1 / 2$.

In physical applications of the coupled system (1) and (2) as discussed in the appendix, the presence of instabilities indicates that more terms need to be included in the model. However, in the stable cases the model is indeed expected to be physically meaningful. And in this regard, the two integrable cases can provide useful starting points for perturbation theory.

As a final remark, let us indicate the kind of calculations that are possible for the coupled system (1) and (2) for $\kappa=1 / 2$ with the aid of the completeness relation. For a family of relevant initial data for the linear equation, one can explicitly compute the projection onto the bound states and consequently determine the long time behavior of the corresponding solution of (2). Also, the long time behavior of the dispersive part of the solution can be computed from the explicit representation of this component of the solution as a singular integral. We leave such applications of the completeness relation for further investigations.

Appendix A. Some applications. It is useful to keep in mind some applications in which the coupled system (1) and (2) might arise. In fact, such equations appear in the modeling of coupling of acoustic phonons in long polymer molecules. Many organic polymers (e.g., DNA and $\alpha$-helix proteins like acetanelide) may be considered from the mechanical point of view as long chains of nearly identical masses. This "backbone" of the molecule supports a longitudinal vibrational mode in which the masses are all moving in tandem with zero frequency (i.e., simple translation) in 


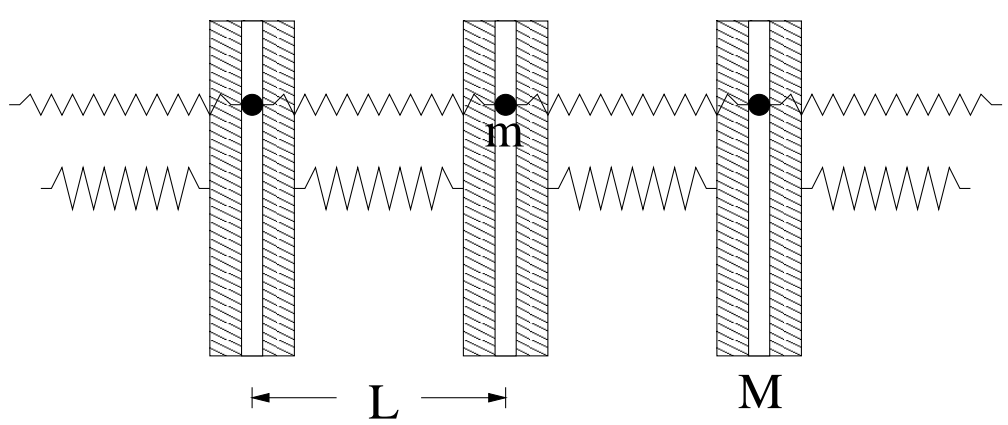

FIG. A.1. The equilibrium configuration of the mechanical model.

the long-wave limit; the associated quanta are called acoustic phonons. In the presence of intrinsic weak nonlinearity, the KdV equation describes these vibrations in the long-wave limit. Usually, the masses making up the chain contain internal degrees of freedom (e.g., the "breathing" modes of base-pairs in DNA, and the so-called amide I exciton modes of the $\mathrm{C}=\mathrm{O}$ bond in each peptide group of an $\alpha$-helix protein). The coupling of these internal degrees of freedom to the motion of the backbone leads to a variety of interesting dynamical models (e.g., the discrete sine-Gordon equation for DNA and the discrete nonlinear Schrödinger equation for $\alpha$-helix proteins).

We may consider a situation in which the internal degrees of freedom are themselves acoustic phonons associated with transverse vibrational modes. This can be visualized with the help of a concrete mechanical model, whose equilibrium configuration is shown in Figure A.1. The backbone is made of heavy masses $M$ connected by stiff springs. Mounted on each heavy mass is a transversely-oriented frictionless track in which rides a small mass $m$. The mass $M$ is assumed to include the mass of the track and small mass $m$. The small masses are themselves connected by weaker springs. Assigning longitudinal displacements $u_{n}$ to the large masses $M$ and transverse displacements $v_{n}$ to the small masses $m$ in the frictionless tracks, the Hamiltonian of the mechanical model is

$$
\begin{gathered}
H=\sum_{n}\left[\frac{1}{2} M \dot{u}_{n}^{2}+\frac{1}{2} m \dot{v}_{n}^{2}+W\left(L+u_{n+1}-u_{n}\right)\right. \\
\left.+V\left(\sqrt{\left(L+u_{n+1}-u_{n}\right)^{2}+\left(v_{n+1}-v_{n}\right)^{2}}\right)\right],
\end{gathered}
$$

(A1)

where $W$ is the potential energy of the stiff springs connecting the large masses and $V$ is the potential energy of the weaker springs.

The associated equations of motion are

$$
\begin{aligned}
M \ddot{u}_{n}= & W^{\prime}\left(L+u_{n+1}-u_{n}\right)-W^{\prime}\left(L+u_{n}-u_{n-1}\right) \\
& +S\left(D_{n+1}\right)\left(L+u_{n+1}-u_{n}\right)-S\left(D_{n}\right)\left(L+u_{n}-u_{n-1}\right), \\
m \ddot{v}_{n}= & S\left(D_{n+1}\right)\left(v_{n+1}-v_{n}\right)-S\left(D_{n}\right)\left(v_{n}-v_{n-1}\right),
\end{aligned}
$$

where we have set $S(D):=V^{\prime}(D) / D$ and $D_{n}:=\sqrt{\left(L+u_{n}-u_{n-1}\right)^{2}+\left(v_{n}-v_{n-1}\right)^{2}}$. 
It is clear that one may take the undisturbed state of the internal modes $v_{n+1}=v_{n}$ for all $n$ to hold exactly, in which case only the backbone motion is relevant. We will be interested in small amplitude, linear motions of the $v_{n}$, and how they are affected by the motion of the backbone.

The disparity between the masses $M$ and $m$, and that of the strengths of the associated springs, is introduced by letting $\mu$ be a small parameter and assuming $m=\mu M$ and $V=\mu U$ (and correspondingly, $S=\mu Z$ ). We make the small-amplitude long-wave ansatz

$$
u_{n}(t)=h u(X=h n, T=h t) \quad \text { and } \quad v_{n}(t)=h v(X=h n, T=h t),
$$

(A3)

where $h$ is a small lattice-spacing parameter. Expanding the functions $W$ and $Z$ in Taylor series about the equilibrium position, the equations of motion become

$$
\begin{aligned}
M h^{3} \partial_{T}^{2} u= & h^{3} W^{\prime \prime}(L) \partial_{X}^{2} u+\frac{h^{5}}{12} W^{\prime \prime}(L) \partial_{X}^{4} u \\
& +h^{5} W^{\prime \prime \prime}(L) \partial_{X} u \cdot \partial_{X}^{2} u+O\left(h^{6}\right)+O\left(h^{3} \mu\right), \\
M h^{3} \partial_{T}^{2} v= & h^{3} Z(L) \partial_{X}^{2} v+\frac{h^{5}}{12} Z(L) \partial_{X}^{4} v \\
& +h^{5} Z^{\prime}(L) \partial_{X}^{2} u \cdot \partial_{X} v+h^{5} Z^{\prime}(L) \partial_{X} u \cdot \partial_{X}^{2} v+O\left(h^{6}\right) .
\end{aligned}
$$

(A4)

Trapping of $v$-waves by $u$-waves becomes possible if the wave speeds are equal. Therefore, we assume that $W^{\prime \prime}(L)=Z(L)=M c^{2}$. Changing variables to $\chi=X-c T$ and $\tau=h^{2} T$ yields

$$
\begin{aligned}
M h^{2} \partial_{\tau}^{2} u-2 M c \partial_{\chi} \partial_{\tau} u & =\frac{M c^{2}}{12} \partial_{\chi}^{4} u+W^{\prime \prime \prime}(L) \partial_{\chi} u \cdot \partial_{\chi}^{2} u+O(h)+O\left(\mu / h^{2}\right) \\
M h^{2} \partial_{\tau}^{2} v-2 M c \partial_{\chi} \partial_{\tau} v & =\frac{M c^{2}}{12} \partial_{\chi}^{4} v+Z^{\prime}(L) \partial_{\chi}^{2} u \cdot \partial_{\chi} v+Z^{\prime}(L) \partial_{\chi} u \cdot \partial_{\chi}^{2} v+O(h) .
\end{aligned}
$$

(A5)

As $h \downarrow 0$ with $\mu \ll h^{2}$, we find the coupled system

(A6)

$$
\begin{aligned}
& \partial_{\tau} A+\partial_{\chi}\left[\frac{1}{2} A^{2}+\frac{c}{24} \partial_{\chi}^{2} A\right]=0 \\
& \partial_{\tau} B+\partial_{\chi}\left[\frac{Z^{\prime}(L)}{W^{\prime \prime \prime}(L)} A B+\frac{c}{24} \partial_{\chi}^{2} B\right]=0
\end{aligned}
$$

as a formal limit, where $A=W^{\prime \prime \prime}(L) \partial_{\chi} u /(2 M c)$ and $B=\partial_{\chi} v$. After a simple rescaling of $\chi$ and $\tau$, this becomes (1) and (2) with $\kappa=Z^{\prime}(L) / W^{\prime \prime \prime}(L)$. As described in section 6 , the influence of solitons on linear waves can be qualitatively different for different values of the coupling constant $\kappa$ with important bifurcations occurring at the values $\kappa=\kappa_{n}^{\text {bif }}=(n+1)(n+2) / 12$ for $n=1,2,3, \ldots$. As we have seen, this coupled system can be solved exactly in (at least) two cases: $\kappa=1$ and $\kappa=1 / 2$. The former case is just the linearized KdV; see Sachs [S83]. The latter case is the one that is solved in the main text of this paper.

Consider this example with the potential of the strong and weak springs given respectively by 
(A7)

$$
W(D):=\frac{1}{2} \kappa_{w} D^{2}+\frac{1}{24} \alpha D^{4} \quad \text { and } \quad V(D):=\mu\left(\frac{1}{2} \kappa_{v} D^{2}+\frac{1}{24} \beta D^{4}\right) .
$$

Thus $\beta=3 \alpha \kappa$ and the condition that the wave speeds are equal is

$$
\frac{1}{2} \alpha L^{2}(\kappa-1)=\kappa_{w}-\kappa_{v} .
$$

The effect of each bifurcation point in $\kappa$ is now clear. At $\kappa=1 / 2$ the first harmonic of the $v$-waves begins to resonate with the $u$-waves. As $\kappa$ increases through $\kappa=1$ we pass through a transition from supercritical resonance to subcritical resonance. Similarly, at the odd bifurcation points, $\kappa=\kappa_{2 m-1}^{\text {bif }}$ for $m=1,2,3, \ldots$, the $m$ th harmonic $v$-wave begins to resonate with the $u$-waves. Then at the even bifurcation points, $\kappa=\kappa_{2 m}^{\text {bif }}$ for $m=1,2,3, \ldots$, the nature of the resonance for this mode changes from supercritical to subcritical.

Coupled systems of equations like the pair (1) and (2) often arise as formal asymptotic reductions of mechanical models for complicated one-dimensional waves. Often these asymptotic models are integrable. For example, in an elastic rod, the interaction between axial twist waves and helical deformation waves gives rise to an integrable Manakov system of coupled nonlinear Schrödinger equations [LG99].

The coupled system (1) and (2) for $\kappa=1 / 2$ is also intimately connected with an integrable multicomponent (an arbitrary number of components, all appearing symmetrically) coupled KdV equation [MC99]. Indeed, from one point of view it is this connection that yields the solvability of (1) and (2) for $\kappa=1 / 2$ described in detail in this paper. The solution method presented here also can be used to give the complete solution of the coupled KdV system. That system in turn can be interpreted as a phenomenological model for the transport of the mass integral through an $N$ soliton solution of KdV [MC99].

Finally, we would like to point out that there are also some applications in which linear equations of the form (2) occur with $\kappa A(x, t)$ being a given function. In this case, $c(x, t)=\kappa A(x, t)$ represents a given spatiotemporal modulation of the speed of linear dispersive waves, say, due to propagation in an inhomogeneous medium. Such problems arise in the modeling of the propagation of weak internal waves in a channel of varying width [CG99]. For such applications, we may view the solvability of the coupled system (1) and (2) for $\kappa=1$ and $\kappa=1 / 2$ as a kind of (big) catalog of special cases of the function $c(x, t)$ for which the linear equation $(2)$ is solvable in its own right. For other values of $\kappa>1 / 2$ the linear wave system is unstable, while for all values of $\kappa<1 / 2$ it is stable.

\section{REFERENCES}

[CG99] S. R. Clarke ANd R. H. J. Grimshaw, Weakly nonlinear internal wave fronts trapped in contractions, J. Fluid Mech., 415 (2000), pp. 323-345.

[ChQZ88] C. Canuto, M. Hussaini, A. Quateroni, And T. Zang, Spectral Methods in Fluid Dynamics, Springer-Verlag, Berlin, 1988.

[GGKM67] C. Gardner, J. Greene, M. Kruskal, and R. Miura, Method for solving the Korteweg-de Vries equation, Phys. Rev. Lett., 19 (1967), pp. 1095-1097.

[GGKM74] C. Gardner, J. Greene, M. Kruskal, and R. Miura, Korteweg-de Vries equation and generalizations, VI. Methods for exact solution, Comm. Pure Appl. Math., 27 (1974), pp. 97-133.

[HS81] R. Hirota AND J. SATSUma, Soliton solutions of a coupled Korteweg-de Vries equation, Phys. Lett., 85A (1981), pp. 407-408. 
[KM56] I. KAY AND H. E. MosEs, Reflectionless transmission through dielectrics and scattering potentials, J. Appl. Phys., 27 (1956), pp.1503-1508.

[LG99] J. Lega AND A. Goriely, Pulses, fronts and oscillations of an elastic rod, Phys. D, 132 (1999), pp. 373-391.

[MA98] P. D. Miller AND N. N. Akhmediev, Modal expansions and completeness relations for some time-dependent Schrödinger equations, Phys. D, 123 (1998), pp. 513-524.

[MC99] P. D. Miller And P. L. Christiansen, A coupled Korteweg-de Vries system and mass exchanges among solitons, Phys. Scripta, 61 (2000), pp. 518-525.

[S83] R. L. SACHS, Completeness of derivatives of squared Schrödinger eigenfunctions and explicit solutions of the linearized KdV equation, SIAM J. Math. Anal., 14 (1983), pp. $674-683$. 